\title{
Problemas Comportamentais em Crianças Pré-Escolares com Fissura Labiopalatina
}

\author{
Francislaine da Silva ${ }^{\mathbf{1}}$ \\ Programa de Pós-Graduação em Psicologia do Desenvolvimento e Aprendizagem \\ da Universidade Estadual “Júlio de Mesquita Filho”, Bauru, SP, Brasil \\ Olga Maria Piazentin Rolim Rodrigues \\ Departamento de Pós-Graduação em Psicologia do Desenvolvimento e Aprendizagem \\ da Universidade Estadual “Júlio de Mesquita Filho”, Bauru, SP, Brasil \\ José Roberto Pereira Lauris \\ Departamento de Odontopediatria, Ortodontia e Saúde Coletiva da Universidade \\ de São Paulo, Bauru, SP, Brasil
}

\begin{abstract}
Resumo
O presente estudo objetivou identificar problemas de comportamento de 83 crianças com fissura labiopalatina e testar a correlação entre esses problemas e as variáveis sexo, idade e tipo de fissura.Os instrumentos utilizados foram: Protocolo de Entrevista e o CBCL.Procedeu-se a análise descritiva dos dados utilizando medidas de frequência e porcentagem e,para as associações, foi realizado o Teste Estatístico do Qui-quadrado. Os resultados mostraram maior frequência de problemas de comportamento externalizantes em meninos. Verificou-se porcentagem maior de problemas internalizantes $(62,2 \%)$ e problemas totais $(46,7 \%)$ em crianças mais velhas. Quanto à relação entre tipo de fissura e problemas comportamentais pode-se constatar a prevalência de comportamentos internalizantes, independentemente do tipo. Considerando o perfil comportamental que compõem as escalas internalizantes e externalizantes, foi observada uma associação entre sexo e comportamento agressivo, com destaque para os meninos; idade e comportamento agressivo, com destaque para 3 a 4 anos e 3 meses e, tipo de fissura pré-forame e transforame e comportamento internalizante, com destaque para os problemas de ansiedade/depressão (50\% e $27,1 \%$ respectivamente). Considera-se que tais dados podem contribuir para o planejamento de ações preventivas e interventivas, uma vez que comportamentos internalizantes e externalizantes reduzem as oportunidades de interações adequadas e, consequentemente, de desenvolvimento social.
\end{abstract}

Palavras-chave: Comportamento infantil, problema de comportamento, fissura labiopalatina.

\section{Behavioral Problems in Preschool Children with Orofacial Clefts}

\begin{abstract}
The present study aimed to evaluate behavioral problems in 83 children with orofacial clefts and to test for possible correlations between these problems and the following variables: gender, age and cleft type. The instruments used were: Interview Protocol and the CBCL. Descriptive analysis of the data was performed using measures of frequency and percentage and the Chi-squared test was used to test for possible correlations. The results showed a higher frequency of externalizing behavior problems in
\end{abstract}

Endereço para correspondência: Departamento de Pós-Graduação em Psicologia do Desenvolvimento e Aprendizagem, Av. Eng. Luiz Edmundo Carrijo Coube, 1401, Vargem Limpa, Bauru, SP, Brasil 17033-360. Fone: (14)3103-6000.E-mail: franlorca24@yahoo.com.bre olgarolim@fc.unesp.br 
boys. Higher rates of internalizing problems $(62.2 \%)$ and total problems $(46.7 \%)$ were also found in older children. Regarding the relationship between cleft type and behavioral problems, a prevalence of internalizing behaviors was observed, regardless of the type. Considering the behavioral profile that composes the internalizing and externalizing scales, an association between male gender and aggressive behavior was observed; between the 3 years to 4 years 3 months age group and aggressive behavior; and between the pre-incisive foramen cleft and trans-incisive foramen cleft types and internalizing behavior, with emphasis on anxiety/depression problems (50\% and $27.1 \%$ respectively). It is considered that these data can contribute to the planning of preventive and interventional actions, as internalizing and externalizing behaviors reduce the opportunity for adequate interactions and, hence social development.

Keywords: Children's behavior, behavior problems, cleft lip and palate.

\section{Problemas de Conducta en Niños Preescolares con Fisuralabiopalatina}

\section{Resumen}

Este estúdio objetivó identificar los problemas de comportamiento de 83 niños con fisura de lábio y paladar e probar la correlación entre estos problemas y el género, la edad y el tipo de fisura. Los instrumentos utilizados fueron: Protocolo de Entrevista y el CBCL. Se procedió al análisis descriptivo de los datos utilizando medidas de frecuencia y porcentaje, y para las asociaciones, se realizó la prueba estadística de Chi-cuadrado. Los resultados mostraron una mayor frecuencia de problemas de comportamiento exteriorizado en los niños. Se encontró un mayor porcentaje de problemas de internalización $(62.2 \%)$ y problemas totales $(46.7 \%)$ en niños de más edad. La relación entre el tipo de fisura y problemas de comportamiento puede observar la prevalencia de conductas de internalización, independientemente del tipo. Teniendo en cuenta el perfil de comportamiento presentes en escalas de internalización y externalización, se observó una asociación entre el sexo y el comportamiento agresivo, especialmente para los niños; la edad y el comportamiento agresivo, especialmente para 3 a 4 años y 3 meses y, el tipo de fisura pre-foramen y transforamen y comportamiento de internalización, poniendo de relieve problemas de ansiedad / depresión (50\% y 27.1\%, respectivamente). Se considera que estos datos pueden contribuir al desarrollo de acciones preventivas y de intervención, ya que los comportamientos de internalización y externalización reducen las oportunidades de interacción adecuados y, por tanto, el desarrollo social.

Palabras clave: Comportamiento infantil, los problemas de conducta, fissura de labio y paladar.

As fissuras labiopalatinas são malformações congênitas que acometem as estruturas da face e do crânio durante o período embrionário e o início do período fetal, sendo representadas, clinicamente, pela ruptura do lábio, palato ou ambos (Silva \& Souza-Freitas, 2007). As fissuras podem apresentar graus variados de gravidade de acordo com sua extensão, podendo ser uni ou bilaterais, completas ou incompletas. A classificação atual toma como ponto de referência o forame incisivo, a fissura que envolve o lábio é denominada de pré-forame incisivo, a fissura de palato, pós-forame incisivo e, a fissura que envolve ambas as estruturas, é denominada de transforame incisivo (Spina, Psillakis, Lapa, \& Ferreira, 1972). Sua etiologia pode ser atribuída a fatores genéticos e ambientais que podem atuar separadamente ou em conjunto (Paranaíba et al., 2010).

Várias são as consequências decorrentes das fissuras labiopalatinas, pois além dos aspectos estéticos, seus portadores são suscetíveis às dificuldades relacionadas à mastigação, à deglutição, à audição, à respiração, aos comprometimentos da arcada dentária, a constantes infecções otorrinolaringológicas, e a distúrbios fonoarticulatórios (voz nasalizada; Paranaíba et al., 2010). 
A fissura labiopalatina também gera consequências de ordem emocional e psicossocial (Lemos \& Feniman, 2010; Oliveira, 2014; Pazinato et al., 2011). Essas contingências produzem consequências que implicam em uma autoestima prejudicada, mais dependência dos pais, isolamento e esquivas de contatos sociais e até redução da capacidade verbal (Domingues, Picolini, Lauris, \& Maximino, 2011; Oliveira, 2014). Diante das consequências da dificuldade de uma comunicação efetiva e da aparência física comprometida, o indivíduo portador de fissura labiopalatina, torna-se alvo de situações marcantes, tais como o preconceito social, a discriminação e a ridicularização que podem comprometer seu desenvolvimento e desempenho social (Guimarães, 2010; Maggi \& Scopel, 2011).

Durante os primeiros anos de vida, a criança vivencia uma série de experiências no seu ambiente familiar e escolar que podem favorecer o desenvolvimento de competência interpessoal ou problemas de comportamento (Borsa \& Nunes, 2011). Os problemas de comportamento referem-se aos excessos ou aos déficits comportamentais que prejudicam, não só o próprio indivíduo que os apresenta, mas, também, a interação da criança com os pares e adultos de sua convivência (Bolsoni-Silva \& Marturano, 2010; Del Prette \& Del Prette, 2013).

Pesquisas têm apontado para uma diminuição da competência social de crianças com fissura labiopalatina, que se caracterizam pelo menor número de amigos, menos contatos sociais e pior qualidade de interações sociais, bem como problemas comportamentais de internalização e externalização (Murray et al., 2010; Snyder \& Pope, 2010). Todavia, as crianças com fissura labiopalatina não se percebem diferentes das outras crianças até a idade de quatro ou cinco anos, especialmente se tiveram uma boa aceitação familiar (Silva, Locks, Carcereri, \& Silva, 2013). Contudo, ao ampliar seu meio social, essa diferença é apontada, podendo acarretar desajustamentos de comportamento, de personalidade e de desempenho social, inclusive o abandono escolar (Maggi \& Scopel, 2011).

A aparência imperfeita pode levar a respostas sociais não favoráveis, consequências de es- tigmas (Silva et al., 2013). A fala comprometida produz angústia, pelo fato de não ser entendida, dando a impressão de limitações intelectuais, físicas e inabilidade social (Guimarães, 2010; Ha, Zhuge, Zheng, Shi, Gong, \& Wang, 2013; Lemos \& Feniman, 2010; Maggi \& Scopel, 2011; Pazinato et al., 2011). Além dos apelidos, as crianças com malformações são olhadas com curiosidade, preconceito e apresentam dificuldades de novos contatos sociais, integração e ajustamento (Miguel, Locks, \& Prado, 2009).

Desta forma, estudos voltados para as relações interpessoais da criança portadora de fissura labiopalatina são importantes, especialmente no início de suas atividades de estudo, que se dá por ocasião do seu ingresso na pré-escola. Para a criança portadora de deformidade facial, será a primeira e a mais importante experiência sistemática fora do ambiente do lar (Graciano, Tavano, \& Bachega, 2007). Neste momento, a criança sai do ambiente seguro e protegido da sua família para enfrentar um ambiente desconhecido, que apresenta exigências e regras diversificadas, inclusive de interação com pessoas até então desconhecidas, para o qual não apresenta todas as habilidades sociais necessárias para enfrentar com eficiência o ambiente escolar (Buffa, 2009).

$\mathrm{O}$ ambiente escolar encontrado pelas crianças portadoras de fissura labiopalatina, torna-se um novo desafio, pois terá de enfrentar novos relacionamentos, será olhada, julgada, avaliada em sua aparência física, bem como, em sua comunicação, variáveis importantes nestes julgamentos (Graciano, Tavano, \& Bachega, 2007; Miguel, Locks, \& Prado, 2009). Como consequência, esse ambiente oferece condições que podem gerar comportamentos de timidez, recolhimento ou agressividade, as quais, ao ser percebida pela criança podem influir no seu desempenho socioemocional (Guimarães, 2010; Maggi \& Scopel, 2011; Thamilselvan, Kumar, Murthy, Sharma, \& Kumar, 2015).

$\mathrm{Na}$ direção de tais achados, Murray et al. (2010) avaliaram o funcionamento socioemocional de crianças com fissura em idade escolar, por meio de observações diretas das interações sociais e representações da criança, bem como por relatórios de mães e de professores. Cons- 
tataram que as crianças apresentaram risco aumentado para as dificuldades sócio-emocionais do ano escolar. Nos relatos dos professores houve indicativos para comportamentos ansiosos, introspectivos e deprimidos e, observações diretas revelaram dificuldades nas interações sociais, principalmente problemas de comunicação.

Dados semelhantes foram encontrados na pesquisa de Richman e Millard (1997), que constataram aumento nos níveis de inibição social sobre a idade para as meninas e discreta elevação consistente no comportamento internalizantes dos meninos. Identificaram também, o aumento dos níveis de problemas de conduta para as meninas mais velhas com fissura, com flutuação de idade variável para os meninos.

Numa revisão sistemática da literatura sobre os efeitos psicossociais em crianças e adultos com fissura labiopalatina, Hunt, Burden, Hepper e Johnston (2005) encontraram dificuldades em classificar o que seriam problemas psicológicos e sociais. Na análise dos dados, destacaram que, em sua maioria, crianças e adultos com fissura não parecem ter mais problemas psicossociais em relação às pessoas sem malformação, embora alguns problemas específicos tenham sido observados como problemas de comportamento, insatisfação com a aparência facial, depressão e ansiedade. Considerando os diferentes tipos de fissura, poucas diferenças foram encontradas em relação ao autoconceito, nível de satisfação com aparência facial, depressão, problemas de aprendizagem e relacionamentos interpessoais. Com poucas exceções, a idade dos sujeitos com fissura pareceu não influenciar na ocorrência ou severidade de problemas psicossociais relativos a esta condição.

Problemas de ajustamento psicossocial e competências sociais foram investigados por Pope e Snyder (2005), em uma amostra de 724 crianças com anomalias congênitas craniofaciais com idade entre 2 e 18 anos, por meio do Child Behavior Checklist (CBCL), desenvolvido por Achenbach e Rescorla (2001). Constataram pontuação clínica para problemas de isolamento em crianças de 2 a 3 anos de idade. Identificaram na faixa etária de 4 a 11 anos maiores taxas de problemas como a introversão, déficit de atenção e problemas internalizantes. Os meninos na faixa etária de 4 a 11 anos apresentaram pontuação clínica para competência escolar e total, enquanto que as meninas pontuaram mais problemas clínicos nas escalas de problemas sociais, déficit de atenção e competência social.

Em outro estudo, Snyder e Pope (2010) investigaram padrões de ajustamento psicossocial em crianças e adolescentes entre 2 e 18 anos de idade com o diagnóstico de fissura labiopalatina, por meio do CBCL. Os autores constataram pontuações significativas para problemas sociais e déficit nas competências sociais e acadêmicas. Identificaram pontuação clínica para problemas externalizantes e para total de problemas de comportamento em crianças de 2 a 3 anos de idade com fissura de lábio e palato. Nas crianças de 4 a 11 anos de idade constataram pontuação clínica para os problemas sociais, competência social e total de problemas de comportamento. As crianças que apresentavam apenas fissura de palato, na faixa etária de 4 a 11 anos mostraram problemas sociais com pontuação clínica.

Ao se analisar a literatura, considerando-se a criança com fissura labiopalatina, na faixa etária entre 3 e 5 anos, observa-se escassez de estudos nacionais e internacionais que investigam problemas de comportamento em tal população. Neste contexto, justifica-se a relevância social e empírica do presente trabalho que pretende contribuir para a identificação e a compreensão do perfil comportamental de crianças com fissura labiopalatina. No que se refere aos aspectos teóricos, significaria preencher a lacuna existente na literatura sobre o assunto.

Com base na literatura, o presente estudo tem por hipóteses: (a) os índices de problemas de comportamento para a amostra deste estudo serão superiores à média geral da amostra estudada no próprio teste; (b) um número maior de problemas comportamentais será registrado para crianças com fissura transforame incisivo, considerada mais grave, do que para crianças com fissura pré-forame e pós-forame incisivo; (c) para os meninos serão identificados mais problemas comportamentais externalizantes e, para as meninas, mais problemas de comportamentos internalizantes e, (d) as crianças mais velhas apresen- 
tarão um aumento dos níveis de problemas de comportamento quando comparadas às crianças mais novas.

Considerando o exposto, o presente estudo objetivou identificar problemas de comportamento de 83 crianças com fissura labiopalatina e testar a correlação entre esses problemas e as variáveis sexo, idade e tipo de fissura. Enquanto objetivos específicos pretendeu-se: (a) identificar os problemas de comportamento (internalizantes e externalizantes) em crianças pré-escolares com fissura labiopalatina, conforme relato de suas mães; (b) testar possíveis correlações entre problemas de comportamento (internalizantes e externalizantes) com as variáveis sexo, idade e tipo de fissura.

\section{Método}

\section{Participantes}

Participaram deste estudo 83 crianças com fissura labiopalatina, sendo 48 meninos (57\%) e 35 meninas (42\%). Da amostra $38(45,78 \%)$ tinham de 3 anos a 4 anos e 3 meses e $45(54,21 \%)$ tinham entre 4 anos e 4 meses e 5 anos e 10 meses. Da amostra, $62(74,70 \%)$ crianças frequentavam a escola. Quanto ao tipo de fissura, 37 (44\%) tinham a fissura transforame, $30(36 \%)$ tinham a pós-forame incisivo e $16(19 \%)$ tinham a fissura pré-forame. Todos encontravam-se em processo de tratamento no Hospital de Reabilitação de Anomalias Craniofaciais da Universidade de São Paulo (HRAC/USP), situado na cidade de Bauru, estado de São Paulo.

\section{Instrumentos}

Para caracterizar as condições sociodemográficas foi utilizado um protocolo de entrevista desenvolvido pela pesquisadora, que consistiu de perguntas abertas e fechadas, com informações referentes à identificação da criança e dados dos componentes familiares. Para a identificação dos indicadores de problemas de comportamento das crianças foi utilizado o Child Behavior Checklist (CBCL versão $1 \frac{1}{2}$ a 5 anos). É um instrumento constituído de 99 itens, destinados à avaliação de problemas comportamentais de crianças. A partir do relato dos pais os itens relativos a problemas de comportamento são classificados de acordo com uma escala de três pontos: item falso ou comportamento ausente (escore $=0$ ); item parcialmente verdadeiro ou comportamento às vezes presente $($ escore $=1)$ e item bastante verdadeiro ou comportamento frequentemente presente (escore $=2$ ). Registrados e somados em escalas, fornecem o perfil comportamental da criança. O perfil comportamental é avaliado por meio de sete eixos ou síndromes (Emocionalmente Reativa, Ansiedade e Depressão, Queixas Somáticas, Isolamento, Problemas de Sono, Problemas de Atenção e Comportamento Agressivo), além das escalas de Internalização, Externalização e Total de problemas de comportamento (Achenbach \& Rescorla, 2000). Uma versão eletrônica do CBCL, o ADM, possibilita a conversão dos escores brutos em escores $\mathrm{T}$, baseados na frequência dos itens do CBCL na população americana. Pontos de corte em escores $\mathrm{T}$ determinam as categorias não clínica, limítrofe e clínica para as escalas sociais e comportamentais (Achenbach \& Rescorla, 2000).

\section{Procedimentos}

O presente projeto de pesquisa foi submetido à Comissão de Pesquisa e ao Comitê de Ética em Pesquisa do Hospital de Reabilitação de Anomalias Craniofaciais da Universidade de São Paulo - USP/Bauru e recebeu parecer favorável (processo CEP-CAAE $\mathrm{n}^{\mathrm{o}}$ 05988512.2.0000.5441). $\mathrm{Na}$ pesquisa procurou-se assegurar a integridade física e moral de todas as mães e seus filhos participantes, abrangendo os aspectos éticos considerados essenciais em pesquisas com seres humanos pela Resolução 466/12 do Conselho Nacional de Saúde (CONEP; Ministério da Saúde, 2012).

Para a coleta dos dados foi realizado, junto à Central de Agendamento do Hospital, um levantamento dos pacientes que apresentavam apenas a fissura labiopalatina e que seriam convocados para consulta de rotina ambulatorial no período de setembro a dezembro de 2012. Havia uma previsão inicial de convidar, no mínimo, 120 mães. Todavia, aceitaram participar da pesquisa 83 mães, das 92 convidadas (aquelas que não aceitaram foi por incompatibilidade de horário). 
Por ocasião da consulta os atendimentos para possível participação no projeto foram agendados. No primeiro atendimento as mães foram informadas sobre a pesquisa, seus objetivos e procedimentos. Dirimidas as dúvidas e explicitados seus direitos e deveres foram convidadas a participar voluntariamente da mesma. Em caso de aceite as mães assinaram um termo de livre consentimento.

Inicialmente foi aplicado, de forma individual, o protocolo de entrevista, contendo um roteiro de coleta de dados sociodemográficos. Logo após, seguiu-se com a aplicação do instrumento Child Behavior Checklist (CBCL versão $1 \frac{1}{2}$ a 5 anos). As mães foram informadas que o questionário se referia a uma lista de itens que descrevia comportamentos de crianças. Foi solicitado para que as mesmas pensassem em sua criança nos dois últimos meses até o momento presente e circulassem os itens. O tempo de duração de cada aplicação foi de aproximadamente de quinze minutos.

Para a correção do CBCL foi utilizado o Software Assessment Data Manager 7.0 (ADM 7.0). O programa ADM 7.0 ao corrigir as respostas fornecidas aos itens do CBCL classifica a criança a partir de categorias 'clínica' (escores acima de 70), 'limítrofe' (escores entre 65 a 69) e 'não-clínica' (escores inferiores a 64; Achenbach \& Rescorla, 2000).

\section{Análise dos Dados}

Os dados foram codificados e digitados em planilhas do programa Excel ${ }^{\circledR}$, compondo um banco de dados para serem analisados utilizando o pacote estatístico Statistica Versão 12.0 (Stat Soft Inc., Tulsa, USA).

Inicialmente foram realizadas análises descritivas a fim de caracterizar o perfil dos participantes quanto às características sociodemográficas (sexo e idade) e o tipo de fissura, utilizando medidas de frequência e porcentagem.

Para verificar as associações entre os dados sociodemográficos, tipo de fissura e os problemas de comportamento das crianças avaliados pelo CBCL foi realizado o Teste Estatístico do Qui-quadrado. Consultada a Tabela do $\chi^{2}$, considerou-se o valor crítico para $\alpha$ de $5 \%$ igual a
3,841 e 5,991 . Adotou-se em todas as análises como critério de significância $p<0,05$.

\section{Resultados}

De acordo com o relato das mães ao responder ao CBCL, observou-se que das 83 crianças avaliadas, 47 (56,6\%) apresentam problemas de comportamento internalizantes em nível clínico. Com relação aos externalizantes, 26 (31,3\%) das mães relataram a presença deles, no repertório comportamental de seus filhos, também em nível clínico. Considerando o total de problemas de comportamento, 39 (47\%) revelaram sua presença em nível clínico (Tabela 1).

Quanto às dimensões do DSM avaliadas pelo CBCL, das 83 crianças $41(49,4 \%)$ apresentaram indicadores clínicos para problemas de déficit de atenção e hiperatividade, 40 (48,2\%) para problemas de ansiedade, $33(39,8 \%)$ apresentaram indicadores clínicos para problemas do desenvolvimento, $30(36,1 \%)$ para problemas afetivos e $24(28,9 \%)$ para problema opositor-desafiante. Considerando o perfil comportamental do total das crianças avaliadas por meio dos sete eixos ou síndromes que compõem o CBCL, observou-se que $23(27,7 \%)$ apresentaram indicadores clínicos para 'ansiedade/ depressão', seguidos de $18(21,7 \%)$ com 'problemas de atenção', 16 (19,3\%) 'emocionalmente reativa', 15 (18\%) 'isolado', 14 (16,9\%) com indicadores para 'comportamento agressivo', $13(15,7 \%)$ com 'queixas somáticas' e $7(8,4 \%)$ com 'problemas de sono' (Tabela 1).

$\mathrm{Na}$ Tabela 2, os comportamentos internalizantes em nível clínico ocorreram para 27 $(56,3 \%)$ dos meninos e para $21(60 \%)$ das meninas, enquanto que os externalizantes ocorreram para $18(37,5 \%)$ dos meninos e para $9(25,7 \%)$ das meninas. Quanto aos problemas totais eles estavam mais presentes no comportamento dos meninos $23(47,9 \%)$ do que no das meninas 15 $(42,9 \%)$.

As crianças tinham entre 3 e 5 anos e 10 meses de idade. Elas foram divididas em duas faixas etárias: 3 anos a 4 anos e 3 meses $(n=38)$ e 4 anos e 4 meses em diante $(n=45)$, conforme mostra Tabela 2. O grupo de crianças mais velhas 
Tabela 1

Descrição dos Indicadores de Problemas Comportamentais Avaliados pelo CBCL

\begin{tabular}{|c|c|c|c|c|}
\hline \multirow{3}{*}{$\begin{array}{c}\text { Indicadores } \\
\text { Comportamentais } \\
\text { Problemas de Comportamentos }\end{array}$} & \multicolumn{4}{|c|}{ Frequência } \\
\hline & \multicolumn{2}{|c|}{ Não Clínico } & \multicolumn{2}{|c|}{ Clínico } \\
\hline & $N$ & $\%$ & $n$ & $\%$ \\
\hline Internalizantes & 36 & 43,4 & 47 & 56,6 \\
\hline Externalizantes & 57 & 68,7 & 26 & 31,3 \\
\hline Problemas Totais & 44 & 53 & 39 & 47 \\
\hline Dimensões do DSM & $N$ & $\%$ & $n$ & $\%$ \\
\hline Problemas Afetivos & 53 & 63,9 & 30 & 36,1 \\
\hline Problemas Ansiedade & 43 & 51,8 & 40 & 48,2 \\
\hline Problemas Desenvolvimento & 50 & 60,2 & 33 & 39,8 \\
\hline Problemas Déficit Atenção e Hiperatividade & 42 & 50,6 & 41 & 49,4 \\
\hline Problemas Opositor-Desafiante & 59 & 71,1 & 24 & 28,9 \\
\hline Eixos ou Síndromes & $N$ & $\%$ & $n$ & $\%$ \\
\hline Emocionalmente Reativa & 67 & 80,7 & 16 & 19,3 \\
\hline Ansiedade/Depressão & 60 & 72,3 & 23 & 27,7 \\
\hline Queixas Somáticas & 70 & 84,3 & 13 & 15,7 \\
\hline Isolado & 68 & 82 & 15 & 18 \\
\hline Problemas de Sono & 76 & 91,6 & 7 & 8,4 \\
\hline Problemas de Atenção & 65 & 78,3 & 18 & 21,7 \\
\hline Comportamento Agressivo & 69 & 83,1 & 14 & 16,9 \\
\hline
\end{tabular}

(28 - 62,2\%) apresentou mais comportamentos internalizantes em nível clínico do que as crianças mais novas $(20-52,6 \%)$. As crianças mais novas $(13-34,2 \%)$ apresentaram mais comportamentos externalizantes do que as crianças mais velhas $(14-31,1 \%)$ e as crianças mais velhas $(21$ - 46,7\%) apesentaram mais os problemas totais do que as crianças mais novas (17 - 44,7\%).

Todas as crianças da amostra tinham fissura do forame e, para esta análise, foram divididas em pré-forame $(n=16)$, pós-forame $(n=30)$ e transforame $(n=37)$. Das crianças, $10(62,5 \%)$ das com fissura pré-forame apresentaram comportamentos internalizantes em nível clínico, 21 (70\%) das com fissura pós-forame e 17 (45,9\%) com fissura transforame. Quanto aos comportamentos externalizantes, três $(18,7 \%)$ das crianças com fissura pré-forame os apresentaram, nove
(30\%) das com pós-forame e $15(40,5 \%)$ das crianças com fissura transforame. As crianças com fissura transforame (19 - 51,4\%) apresentaram mais problemas totais do que as crianças com fissura pós-forame (13 - 43,3\%) e do que as crianças com fissura pré-forame $(6-37,5 \%)$ (Tabela 2). Não houve diferença estatisticamente significativa na comparação entre grupos considerando idade da criança, sexo e tipo de fissura.

Os dados relativos às dimensões avaliadas pelo CBCL estão apresentados na Tabela 3. Ela revela maior frequência de problemas de desenvolvimento para ambos os sexos (48 (33,3\%) meninos e 35 (31,4\%) meninas). Considerando os indicadores clínicos para os problemas de ansiedade e déficit de atenção e hiperatividade, $12(25 \%)$ dos meninos os apresentam (respectivamente), enquanto que $9(25,7 \%)$ das meninas 
Tabela 2

Associações dos Comportamentos Internalizantes, Externalizantes e Totais, de Acordo com o Relato das Mães, conforme Sexo, Idade e Tipo de Fissura das Crianças

\begin{tabular}{|c|c|c|c|c|c|c|c|c|c|c|c|c|}
\hline \multirow{2}{*}{ Variáveis } & \multicolumn{4}{|c|}{ Internalizantes } & \multicolumn{4}{|c|}{ Externalizantes } & \multicolumn{4}{|c|}{ Problemas Totais } \\
\hline & Não Clín. & Clín. & $\chi^{2}$ & $p$ & Não Clín. & Clín. & $\chi^{2}$ & $p$ & Não Clín. & Clín. & $\chi^{2}$ & $p$ \\
\hline $\begin{array}{l}\text { Masculino } \\
\quad(n=48)\end{array}$ & $21(43,7)$ & $27(56,3)$ & 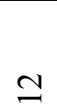 & $m$ & $30(62,5)$ & $18(37,5)$ & $\stackrel{\infty}{\sim}$ & $\begin{array}{l}\infty \\
n\end{array}$ & $25(52,1)$ & $23(47,9)$ & $\vec{\sim}$ & $\stackrel{\infty}{+}$ \\
\hline $\begin{array}{l}\text { Feminino } \\
(n=35)\end{array}$ & $14(40)$ & $21(60)$ & $0^{\circ}$ & $\hat{0}$ & $26(74,3)$ & $9(25,7)$ & & గn & $20(57,1)$ & $15(42,9)$ & & 0 \\
\hline $\begin{array}{c}3 \mathrm{a}-4 \mathrm{a} 3 \mathrm{~m} \\
(n=38)\end{array}$ & $18(47,4)$ & $20(52,6)$ & & & $25(65,8)$ & $13(34,2)$ & & & $21(55,3)$ & $17(44,7)$ & & \\
\hline $\begin{array}{c}4 \mathrm{a} \text { e } 4 \mathrm{~m} \text { em } \\
\text { diante } \\
(n=45)\end{array}$ & $17(37,8)$ & $28(62,2)$ & $\frac{\infty}{0}$ & $\begin{array}{l}\frac{\infty}{n} \\
n_{0}\end{array}$ & $31(68,9)$ & $14(31,1)$ & $\begin{array}{l}8 \\
0 \\
0\end{array}$ & 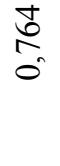 & $24(53,3)$ & $21(46,7)$ & $\stackrel{2}{0}$ & $\begin{array}{l}8 \\
\infty \\
0 \\
0\end{array}$ \\
\hline $\begin{array}{l}\text { Pré-Forame } \\
\quad(n=16)\end{array}$ & $6(37,5)$ & $10(62,5)$ & & & $13(81,3)$ & $3(18,7)$ & & & $10(62,5)$ & $6(37,5)$ & & \\
\hline $\begin{array}{l}\text { Pós-Forame } \\
\qquad(n=30)\end{array}$ & $\begin{array}{c}9 \\
(30)\end{array}$ & $21(70)$ & $\exists$ & $\frac{\infty}{\underset{0}{\sim}}$ & $21(70)$ & $\begin{array}{c}9 \\
(30)\end{array}$ & in & 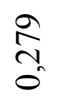 & $17(56,7)$ & $13(43,3)$ & $\stackrel{\infty}{\sigma}$ & $\begin{array}{l} \pm \\
0 \\
0\end{array}$ \\
\hline $\begin{array}{l}\text { Transforame } \\
\qquad(n=37)\end{array}$ & $20(54,1)$ & $17(45,9)$ & & & $22(59,5)$ & $15(40,5)$ & & & $18(48,6)$ & $19(51,4)$ & & \\
\hline
\end{tabular}

apresentam indicadores para problemas de ansiedade e 7 (20\%) para os problemas de déficit de atenção e hiperatividade. Os meninos apresentaram risco para problemas afetivos e opositor-desafiante (9 $(18,7 \%)$, respectivamente), enquanto que $4(11,4 \%)$ das meninas apresentaram (respectivamente) para os mesmos problemas. Entretanto, nota-se também, que não há, para a amostra estudada, associação entre o sexo e às dimensões do DSM avaliadas pelo CBCL.

Considerando a idade das crianças avaliadas, a Tabela 3 mostra que $15(33,3 \%)$ das crianças mais velhas, apresentam indicadores clínicos para problemas de desenvolvimento, enquanto que $12(31,6 \%)$ das crianças mais novas os apresentam. Com relação aos problemas de ansiedade e déficit de atenção e hiperatividade, nota-se que as crianças mais novas apresentam indicadores clínicos $11(28,9 \%)$ e $10(26,3 \%)$, respectivamente), enquanto que $10(22,2 \%)$ e 9 $(20 \%)$, respectivamente das mais velhas os apresentam. No que se refere aos problemas afetivos e opositor-desafiante, $8(17,8 \%)$ e $7(15,6 \%)$, respectivamente das crianças mais velhas apresentam maiores indicadores, enquanto que as mais novas apresentam $5(13,2 \%)$ e $3(7,9 \%)$, respectivamente. Os dados mostram que não há, para a população estudada, associação significativa entre idade e as dimensões do DSM avaliadas pelo CBCL.

A Tabela 3 mostra que $5(31,2 \%)$ das crianças com a fissura pré-forame apresentaram 'problemas de ansiedade' e 'problemas de desenvolvimento', respectivamente, enquanto que 11 $(36,7 \%)$ das crianças com a fissura pós-forame apresentaram 'problemas de desenvolvimento' e 8 (26,7\%) delas, 'déficit de atenção e hiperatividade' e as com fissura transforame, $11(29,8 \%)$ crianças apresentaram 'problemas de desenvolvimento' e 10 (27\%) 'problemas de ansiedade' e 'déficit de atenção e hiperatividade', respectivamente. Todavia, não foram encontradas associações significativas.

Analisando os itens que compõem os problemas de desenvolvimento identificou-se maiores indicativos para as questões referentes a: (1) 
Não responde quando pessoas falam com ela; (2) Fica nervosa com pessoas ou situações novas; (3) Fica perturbada por alguma mudança na rotina; (4) Evita olhar as pessoas nos olhos; e (5) Problema na fala. A informação da questão (1) sugere que as crianças da amostra podem apresentar déficit na atenção auditiva sustentada, como já foi dito anteriormente, o que impossibilita a criança manter o foco atencional, por isto não responde quando pessoas falam com ela. As questões (2) e (3) podem estar relacionadas à ansiedade das crianças frente a situações adversas que deverão enfrentar no seu dia-a-dia. Consi- derando que a maioria das crianças da amostra frequenta a pré-escola e/ou precisa passar por rotinas ambulatoriais e/ou internações para o tratamento da fissura, podemos inferir que o contato com situações ou pessoas novas e a mudança de rotina pode acarretar desajustes de comportamento. O conteúdo retratado na questão (4) pode estar relacionado à própria fissura, sugerindo que essas crianças já se percebem diferentes, ou ainda, por questão da timidez, o que faz com que essas crianças evitam olhar as pessoas nos olhos. Com relação à questão (5) podemos inferir que esteja relacionada às implicações funcionais da fissura labiopalatina.

Tabela 3

Associação das Dimensões do DSM Avaliadas pelo CBCL conforme Sexo, Idade e Tipo de Fissura das Crianças

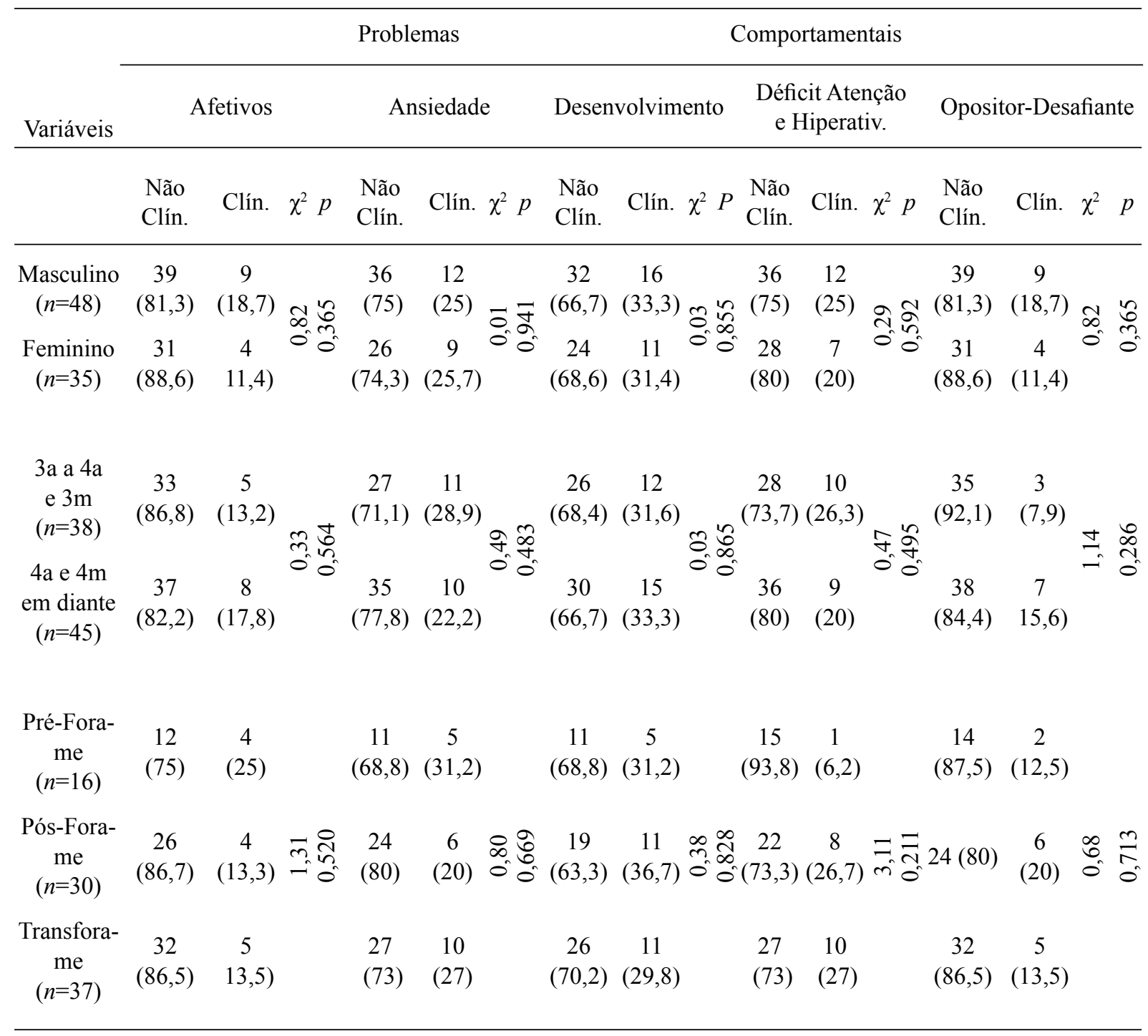


Analisou-se, ainda, os eixos ou síndromes avaliadas pelo CBCL. A Tabela 4 mostra que 16 $(33,3 \%)$ dos meninos apresentam indicadores clínicos para problemas 'ansiedade/depressão' e $12(25 \%)$ para 'comportamento agressivo'. No que se refere às meninas, $8(22,9 \%)$ apresentam indicadores clínicos para 'emocionalmente reativa' e 7 (20\%) para 'ansiedade/depressão' e 'problemas de atenção, respectivamente. Não obstante, os dados mostraram associação estatisticamente significativa para o 'comportamento agressivo' $(p=0,020)$, mais presente entre os meninos. Observou-se, ainda, na Tabela 4 que $11(28,9 \%)$ das crianças mais novas apresentaram indicadores clínicos para problemas 'ansiedade e depressão' e 10 (26,3\%) delas apresentaram indicadores para 'emocionalmente reativa' e 'comportamento agressivo', respectivamente. Enquanto que $12(26,7 \%)$ das crianças mais velhas apresentaram indicadores clínicos para 'ansiedade/depressão' e 9 (20\%) delas indicadores para 'isolado' e 'problemas de atenção', respectivamente. Todavia, os dados mostraram associação significativa para o 'comportamento agressivo' $(p=0,035)$, mais frequente entre as crianças mais novas.

Com relação aos tipos de fissura, nota-se na Tabela 4 que 8 (50\%) das crianças com pré-forame incisivo apresentam indicadores clínicos para problemas 'ansiedade/depressão', enquanto que $4(25 \%)$ apresentaram indicadores para 'emocionalmente reativa'. Das crianças com pós-forame incisivo, $8(26,7 \%)$ apresentaram indicadores clínicos para 'emocionalmente reativa', enquanto que $7(23,3 \%)$ delas apresentaram problemas de 'atenção'. Já as com transforame incisivo, 10 (27,1\%) apresentaram indicadores clínicos para 'ansiedade/depressão' e 9 (24,3\%) delas para 'problemas de atenção'. Entretanto, não há associações significativas entre o tipo de fissura e às síndromes avaliadas pelo CBCL.

\section{Discussão}

Considerando o objetivo do presente estudo que foi identificar por meio do relato das mães, os problemas de comportamento internalizantes e externalizantes em crianças com fis- sura labiopalatina, com idade entre 3 e 5 anos, pode-se constatar a prevalência de comportamentos internalizantes na amostra. Esse achado é apoiado pelo estudo de Murray et al. (2010) que encontrou risco aumentado para as dificuldades socioemocionais em crianças com fissura labiopalatina, com indicativos para comportamentos ansiosos, introspectivos e deprimidos, além de constatarem dificuldades nas interações sociais, típicos de comportamentos internalizantes. Outro estudo que confirma os resultados encontrados é o de Pope e Snyder (2005), que constataram pontuação clínica para problemas de isolamento em crianças com anomalias craniofaciais na faixa etária de 2 a 3 anos de idade e problemas internalizantes, tais como os de introversão, atenção e pensamento na faixa etária de 4 anos de idade.

Com relação as dimensões do DSM avaliadas pelo instrumento, identificou-se na amostra que 49,4\% apresentaram indicadores clínicos para problemas de déficit de atenção e hiperatividade, seguido de indicativos clínicos para problemas de ansiedade (48,2\%). Brand e Brennan (2009) e Wehby et al. (2012) também constataram riscos elevados para problemas de déficit de atenção e hiperatividade em crianças com fissura labiopalatina na idade escolar. No que se refere aos problemas de ansiedade, os dados corroboram os achados de Murray et al. (2010) que identificaram indicadores clínicos para ansiedade em crianças com fissura labiopalatina na idade pré-escolar.

Considerando o perfil comportamental das crianças com fissura labiopalatina avaliadas por meio dos sete eixos ou síndromes que compõem o instrumento CBCL, constatou-se maiores indicativos clínicos para ansiedade/depressão $(27,7 \%)$, seguidos de problemas de atenção $(21,7 \%)$. Esses dados corroboram os achados de Ha et al. (2013), Hunt et al. (2005), Murray et al. (2010) e Pope e Snyder (2005), que identificaram indicativos clínicos para ansiedade/depressão e problemas de atenção em crianças com fissura labiopalatina na idade pré-escolar.

Em relação às associações dos resultados gerais do CBCL (problemas internalizantes, externalizantes e problemas totais) com a variável 


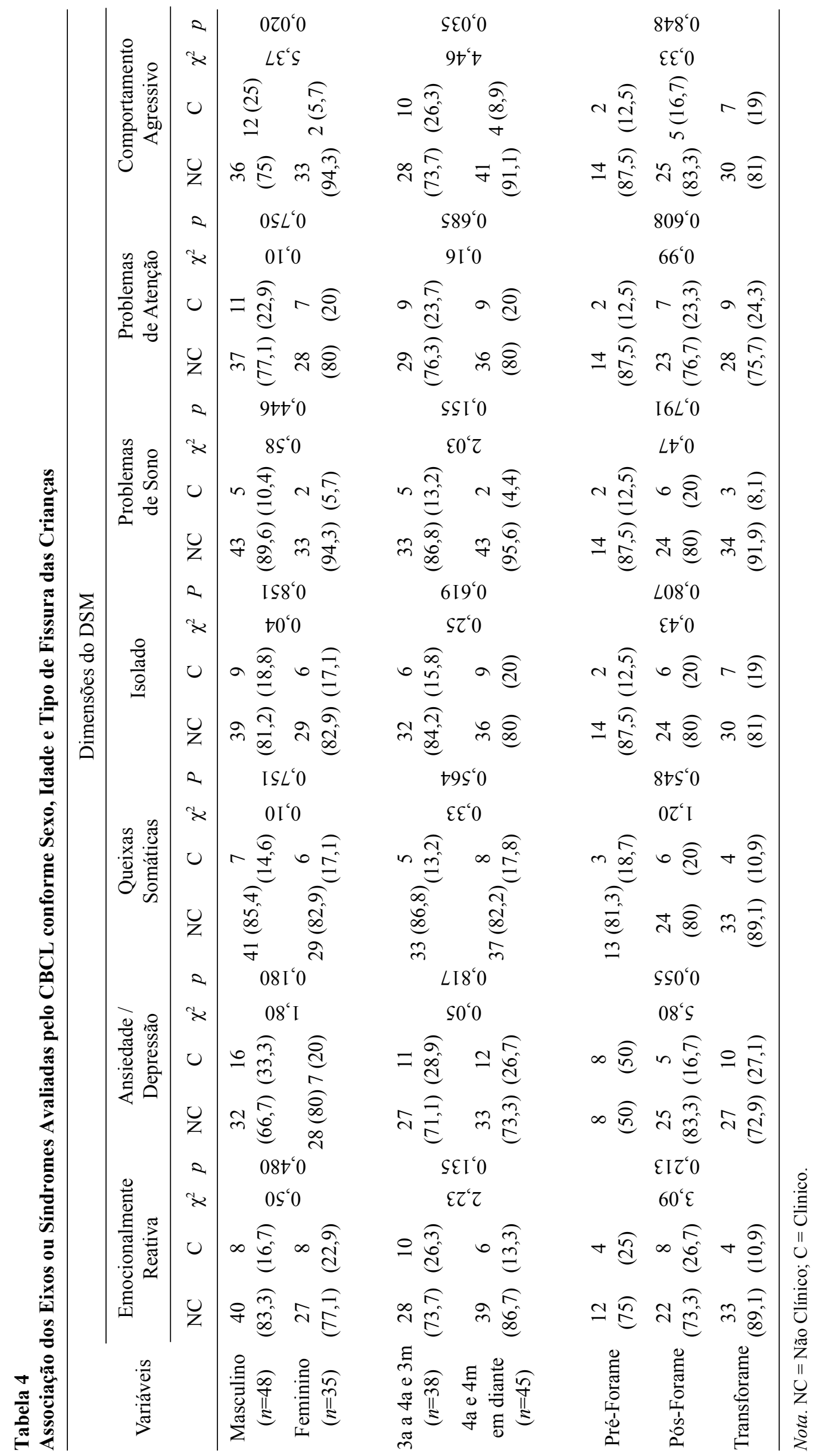


sexo evidenciou-se tanto meninas como meninos apresentaram problemas internalizantes, tal como o encontrado por Richman e Millard (1997). Não obstante, observou-se porcentagem maior de problemas externalizantes e problemas de comportamento totais para os meninos. De maneira geral, tais dados são compatíveis com a literatura quando revela maior frequência de comportamentos externalizantes entre os meninos e internalizantes entre as meninas, também independente da existência da fissura (Blatt-Eisengart et al., 2009; Loosli \& Loureiro, 2010; Nopoulos et al., 2010).

Os dados revelaram, também, que não houve diferenças entre frequência de problemas de comportamento entre crianças mais velhas (4 anos e 4 meses em diante) e mais novas ( 3 anos a 4 anos e 3 meses), diferente do encontrado no estudo de Lins, Alvarenga, Paixão, Almeida e Costa (2012) que constataram maior prevalência de problemas externalizantes em crianças aos 4 anos de idade. De forma semelhante, Pope e Snyder (2005) identificaram altas taxas de problemas internalizantes em crianças com anomalias congênitas aos 4 anos de idade. Todavia, é preciso reconhecer o tamanho reduzido da amostra analisada no presente estudo.

No que se refere às associações dos resultados gerais do CBCL (problemas internalizantes, externalizantes e problemas totais) com a variável tipo de fissura, constatou-se que $70 \%$ das crianças com a fissura do tipo pós-forame apresentaram problemas de comportamento internalizantes, enquanto que $40,5 \%$ e $51,4 \%$ das crianças com a fissura do tipo transforame apresentaram problemas de comportamento externalizantes e problemas totais respectivamente. Os resultados são parcialmente concordantes com os achados de Snyder e Pope (2010) que identificaram pontuação clínica para problemas externalizantes e para total de problemas em crianças de 2 a 3 anos de idade com fissura de lábio e palato. Considerando que alterações nas estruturas do palato, característica dos dois tipos de fissura (pós-forame e transforame), podem acarretar distúrbios de fala específicos como a hipernasalidade e as alterações articulatórias decorrentes do escape de ar nasal durante a produção da fala, podemos inferir que a inteligibilidade na comunicação pode levar a criança ao desajuste psicossocial, como inibição do comportamento, timidez e angústia pelo fato de não ser entendida, típicos de comportamentos internalizantes (Lemos \& Feniman, 2010; Pazinato et al., 2011). Além do comprometimento da fala existe o facial, característico da fissura do tipo transforame que também pode acarretar desajustes de comportamento, como os externalizantes, uma vez que a criança com o defeito facial é olhada, julgada e avaliada em sua aparência física (Graciano, Tavano, \& Bachega, 2007; Guimarães, 2010; Maggi \& Scopel, 2011).

Os resultados do presente estudo não revelaram correlações significativas entre as dimensões do DSM avaliadas pelo CBCL, as variáveis sociodemográficas da criança (sexo e idade) e o tipo de fissura. No entanto, independentemente do sexo, idade e tipo de fissura, em torno de $30 \%$ das crianças participantes apresentaram, segundo relato das mães, problemas de desenvolvimento, quando comparadas às outras dimensões avaliadas. $\mathrm{O}$ dado obtido sugere uma investigação mais aprofundada desses problemas nesta população em estudos futuros.

O presente estudo também testou possíveis relações entre os sete eixos ou síndromes avaliadas pelo CBCL e o sexo das crianças. Observou-se associação significativa apenas para comportamento agressivo com destaque para o sexo masculino. Esse dado parece não diferir em crianças sem problemas de saúde, conforme aponta a literatura (Emerich et al., 2012; Loosli \& Loureiro, 2010). Todavia, os resultados são parcialmente concordantes com os achados de Borsa e Nunes (2011) que identificaram que tanto os meninos quanto as meninas apresentam alta prevalência de problemas de comportamentos agressivos (externalizantes), seguidos por problemas de isolamento (internalizantes). Os autores não encontraram diferença estatisticamente significativa entre meninos e meninas para os diferentes tipos de problemas de comportamento.

Também foi observada associação significativa entre comportamento agressivo e idade das crianças com fissura labiopalatina, com destaque 
para 3 anos a 4 anos e 3 meses. Esse dado parece também não diferir em crianças sem a malformação. Essa afirmação está apoiada em Lins et al. (2012) que destaca os comportamentos externalizantes, como a agressividade, os mais frequentes em pré-escolares, com tendência a diminuir com o avanço da idade, podendo ser considerado como transitório no desenvolvimento normal.

Com relação à associação entre os sete eixos ou síndromes avaliadas pelo CBCL e o tipo de fissura das crianças, não foi constatado nenhum dado significativo. Porém, as crianças com o tipo pré-forame e transforame incisivo apresentaram maior porcentagem para problemas de ansiedade/depressão. Este dado é condizente com os achados de Hunt et al. (2005) que encontraram alguns problemas comportamentais específicos em crianças com fissura labiopalatina, tais como a ansiedade e a depressão, porém, não constataram diferenças significativas quanto aos tipos de fissura. Todavia, alguns estudos constataram que crianças portadoras de fissuras labiopalatinas podem apresentar um maior grau de ansiedade quanto maior forem o grau de deformidade facial, dificuldades de fala e relacionamentos sociais também (Ha et al., 2013; Masnari et al., 2013).

\section{Considerações Finais}

No que se refere à relação entre sexo e problemas comportamentais, a hipótese levantada inicialmente foi confirmada para ambos os sexos, que apresentaram maior frequência de problemas internalizantes em meninas e discreta elevação consistente no comportamento internalizantes e maior incidência de comportamentos externalizantes dos meninos. Considerando o perfil comportamental que compõem as escalas internalizantes e externalizantes, foi observada uma associação entre sexo e comportamento agressivo, com destaque para o sexo masculino, corroborando a hipótese de que os meninos seriam identificados com mais problemas de comportamento externalizantes, porém os resultados não corroboraram com a hipótese da associação do sexo feminino e comportamento internalizante.
Quanto à hipótese levantada de que as crianças com fissura labiopalatina considerada mais grave, do tipo transforame incisivo seriam identificadas com mais problemas comportamentais do que as crianças com fissura pré-forame e pós-forame incisivo, não foi confirmada, uma vez que os resultados mostraram porcentagens maiores de problemas de comportamento em crianças com fissura do tipo pós-forame incisivo, enquanto que as crianças com fissura do tipo transforame incisivo ficaram em segundo lugar quanto às porcentagens de problemas. Ainda, independente do tipo de fissura pode-se constatar a prevalência de comportamentos internalizantes na amostra. Considerando o perfil comportamental que compõem as escalas internalizantes e externalizantes, as crianças com o tipo pré-forame e transforame incisivo apresentaram maior porcentagem para problemas de ansiedade/depressão, confirmando parcialmente a hipótese proposta no presente estudo.

Os dados não confirmaram a hipótese de que mais velhas apresentariam um aumento dos níveis de problemas comportamentais comparadas às crianças mais novas. Observou-se no estudo que as crianças mais velhas apresentaram uma porcentagem maior de problemas internalizantes e problemas totais quando comparado aos problemas externalizantes das crianças mais novas. Entretanto, quando considerado o perfil comportamental que compõem as escalas internalizantes e externalizantes, foi observada uma associação entre idade e comportamento agressivo, com destaque para a idade entre três anos a quatro anos e três meses não corroborando com a hipótese proposta no estudo. Os resultados da presente pesquisa apontam para a necessidade de se elaborar e implantar ações preventivas e interventivas, visando à diminuição de problemas comportamentais (internalizantes e externalizantes), no contexto pré-escolar e, especificamente, para a população de crianças com fissuras labiopalatinas. Entre tais estratégias poderiam ser sugeridas as de implantação de programas de intervenção nas escolas, visando o desenvolvimento de um amplo repertório de habilidades sociais e de programas voltados para os pais, com objetivos de promover habilidades sociais educativas, 
para que atuem na promoção de habilidades sociais de seus filhos, proporcionando aos mesmos uma forma mais satisfatória de lidar com as demandas do seu meio. Ainda, considerando que a população avaliada é atendida por equipe multiprofissional no HRAC, tais aspectos também poderiam ser alvo de programas de intervenção da instituição, tanto no formato de orientação para pais, como em ações dirigidas à própria criança.

Faz-se necessário, ao analisar os achados do presente estudo, destacar algumas limitações inerentes aos aspectos metodológicos utilizados. A primeira delas refere-se ao fato das informações sobre o comportamento infantil terem sido avaliadas pelo relato de uma única fonte, as mães, não sendo incluídas outras fontes de relato tais como professores, pais e cuidadores, o que pode ser questionado. Considerando, também, que algumas crianças podem passar mais tempo com professores, cuidadores ou outro membro da família do que com os pais, principalmente com a mãe, podemos inferir que outro adulto teria um conhecimento maior acerca do funcionamento comportamental da criança. Outra limitação se refere ao instrumento utilizado que, apesar de amplamente reconhecido, não permite a realização de análises funcionais que muito auxiliariam na compreensão da relação entre fissura e problemas internalizantes e externalizantes. A coleta de dados poderia ter sido complementada com instrumentos que permitissem tal análise. Há ainda a escassez de estudos nacionais e internacionais referentes à população estudada. A maioria dos estudos sobre indicativos comportamentais centra-se em crianças em processo típico de desenvolvimento e sem transtornos labiopalatinos com idade escolar, limitando, assim, as comparações. Desta maneira, tornam-se fortes os indicativos de que mais pesquisas sejam realizadas a esse respeito.

\section{Referências}

Achenbach, T. M., \& Rescorla, L. A. (2000). Manual for ASEBA preschool forms \& profiles. Burlington, VT: University of Vermont.

Blatt-Eisengart, I., Drabick, D. A., Monahan, K. C., \& Steinberg, L. (2009). Sex differences in the longitudinal relations among family risk factors and childhood externalizing symptoms. Developmental Psychology, 45(2), 491-502. doi: $10.1037 / \mathrm{a} 0014942$

Bolsoni-Silva, A. T., \& Marturano, E. M. (2010). Relacionamento conjugal, problemas de comportamento e habilidades sociais de pré-escolares. Psicologia: Teoria e Pesquisa, 26(1), 67-75. Recuperado em http://www.scielo.br/pdf/ptp/ v26n1/a09v26n1.pdf

Borsa, J. C., \& Nunes, M. L. T. (2011). Prevalência de problemas de comportamento em uma amostra de crianças em idade escolar da cidade de Porto Alegre. Aletheia, 34, 3246. Recuperado em http://www.redalyc.org/ pdf/1150/115022577004.pdf

Brand, S. R., \& Brennan, P. A. (2009). Impact of antenatal and postpartum maternal mental illness: How are the children? Clinical Obstetrics and Gynecology, 52(3), 441-455. doi:10.1097/ GRF.0b013e3181b52930

Buffa, M. J. M. B. (2009). A inclusão da criança com fissura labiopalatina no ensino regular: Uma visão do professor de classe comum (Tese de doutorado, Hospital de Reabilitação de Anomalias Craniofaciais, Universidade de São Paulo, Bauru, SP, Brasil).

Del Prette, Z. A. P., \& Del Prette, A. (2013). Psicologia das habilidades sociais na infância: Teoria e prática. Petrópolis, RJ: Vozes.

Domingues, A. B. C., Picolini, M. M., Lauris, J. R. P., \& Maximino, L. P. (2011). Desempenho escolar de alunos com fissura labiopalatina no julgamento de seus professores. Revista da Sociedade Brasileira de Fonoaudiologia, 16(3), 310-316. doi:10.1590/S1516-80342011000300012

Emerich, D. R., Rocha, M. M., Silvares, E. F. M., \& Gonçalves, J. P. (2012). Diferenças quanto ao gênero entre escolares brasileiros avaliados pelo inventário de comportamentos para crianças e adolescentes (CBCL/6-18). Psico, 43(3), 380-387. Recuperado em http://revistaseletronicas.pucrs.br/ojs/index.php/revistapsico/article/ view/10053

Graciano, M. I. G., Tavano, L. D'. A., \& Bachega, M. I. (2007). Aspectos psicossociais na reabilitação. In I. E. K. Trindade \& O. G. Silva Filho, Fissuras labiopalatinas: Uma abordagem interdisciplinar (pp. 311-333). São Paulo, SP: Santos. 
Guimarães, A. C. P. C. (2010). Jovens com fissura labiopalatina: Avaliação de saúde mental (Tese de doutorado, Hospital de Reabilitação de Anomalias Craniofaciais, Universidade de São Paulo, Bauru, SP, Brasil).

Ha, P., Zhuge, X., Zheng, Q., Shi, B., Gong, C., \& Wang, Y. (2013). Behavioral pattern in Chinese school-aged children with cleft lip and palate. Asian Pacific Journal of Tropical Medicine, 6(2), 162-166. doi:10.1016/S1995-7645(13)60016-4

Hunt, O., Burden, D., Hepper, P., \& Johnston, C. (2005). The psychosocial effects of cleft lip and palate: A systematic review. European Journal of Orthodontics, 27(3), 274-285. doi:10.1093/ ejo/cji004

Lins, T., Alvarenga, P., Paixão, C., Almeida, E., \& Costa, H. (2012). Problemas externalizantes e agressividade infantil: Uma revisão de estudos brasileiros. Arquivos Brasileiros de Psicologia, 4(3), 57-75. Recuperado em http://pepsic.bvsalud.org/pdf/arbp/v64n3/v64n3a05.pdf

Loosli, L., \& Loureiro, S. R. (2010). Associação entre depressão materna e diferenças de gênero no comportamento de crianças: Uma revisão sistemática. Revista de Psiquiatria do Rio Grande do Sul, 32(3), 94-101. doi:10.1590/S010181082010005000001

Maggi, A., \& Scopel, J. B. (2011). Atendimento aos portadores de fissuras labiais e/ou palatais: Características de um serviço. Aletheia, 34(1), 175 186. Recuperado em http://www.redalyc.org/ pdf/1150/115022577014.pdf

Masnari, O., Shiestl, C., Rössler, J., Gütlein, S. K., Neuhaus, K., Weibel, L. Meuli, M., \& Landolt, M. A. (2013). Stigmatization predicts psychological adjustment and quality of life in children and adolescents with a facial difference. Journal of Pediatric Psychology, 38(2), 162-72. doi:10.1093/jpepsy/jss106.

Miguel, L. C. M., Locks, A., \& Prado, M. L. (2009). $\mathrm{O}$ relato das mães quando do início escolar de seus filhos portadores da má-formação labiopalatal. Revista Sul-Brasileira de Odontologia, 6(2), 155-161. Recuperado em http://www.redalyc.org/articulo.oa?id=153013734007

Ministério da Saúde. (2012). Resolução $n^{\circ} 466$, de 12 de dezembro de 2012. Recuperado em http://conselho.saude.gov.br/resolucoes/2012/ Reso466.pdf
Murray, L., Arteche, A., Bingley, C., Hentges, F., Bishop, D. H., Goodacre, T., \& Hill, J. (2010). The effect of cleft on sócio-emotional functioning in school-aged children. Journal of Child Psychology and Psychiatry and Allied Disciplines, 51(1), 94-103. doi:10.1111/j.14697610.2009.02186.x

Nopoulos, P., Boes, A. D., Jabines, A., Conrad, A. L., Canady, J., Richman, L., \& Dawson J.D. (2010). Hyperactivity, impulsivity, and inattention in boys with cleft lip and palate: Relationship to ventromedial pré-frontal cortex morphology. Journal of Neurodevelopmental Disorders, 2(4), 235-242. doi:10.1007/s1 1689-010-9060-5

Oliveira, R. M. R. (2014). Uma abordagem sobre as dificuldades enfrentadas por mães na amamentação de crianças portadoras de fissuras labiopalatinas. Revista Brasileira de Educação e Saúde REBES, 4(2), 1-6. Recuperado em http:/www. gvaa.com.br/revista/index.php/REBES/article/ view/3017

Paranaíba, L. M. R., Miranda, R. T., Martelli, D. R. B., Bonan, P. R. F., Almeida, H., Orsi Júnior, J. M., \& Martelli, H., Jr. (2010). Cleft lip and palate: Series of unusual clinical cases. Brazilian Journal of Otorhinolaryngology, 76(5), 649653. doi:10.1590/S1808-86942010000500019

Pazinato, L. V., Bonow, M. P., Moraes, R. F. P., Liebl, S., Suetugo, R., Pereira, P. P. A., ...Maluf, E. M. C. P. (2011). Qualidade de vida de crianças e adolescentes portadoras de fissura labiopalatal na visão dos cuidadores. Revista da Sociedade Brasileira de Cirurgia Craniomaxilofacial, 14(4), 194-197. Recuperado em http://www.abccmf.org.br/cmf/Revi/2011/out-dez/05-Qualidade\%20de\%20vida\%20 de $\% 20$ crian $\%$ C3\%A7as\%20e\%20adolescentes $\% 20$ portadoras

Pope, A. W. \& Snyder, H. T. (2005). Psychosocial adjustment in children and adolescents with a craniofacial anomaly: Age and sex patterns. Cleft Palate-Craniofacial Journal, 42(4), 349354. doi:10.1597/04-043R.1

Richman, L. C., \& Millard, T. (1997). Brief report: Cleft lip and palate: Longitudinal behavior and relationships of cleft conditions to behavior and achievement. Journal of Pediatric Psychology, 22(4), 487-494. doi:10.1093/jpepsy/22.4.487

Silva, O. G., Filho, \& Souza Freitas, J. A. (2007). Caracterização morfológica e origem embrioló- 
gica. In I. E. K. Trindade \& O. G. Silva-Filho, Fissuras labiopalatinas: Uma abordagem interdisciplinar (pp. 17-49). São Paulo, SP: Santos.

Silva, C. M., Locks, A., Carcereri, D. L., \& Silva, D. G. V. (2013). A escola na promoção da saúde de crianças com fissura labiopalatal. Texto \& Contexto-Enfermagem, 22(4), 1041-1048. doi:10.1590/S0104-07072013000400021

Spina, V., Psillakis, J. M., Lapa, F. S., \& Ferreira, M. C. (1972). Classificação das fissuras lábio-palatais: Sugestão de modificação. Revista do Hospital das Clínicas, 27(1), 5-6.

Snyder, H., \& Pope, A. W. (2010). Psychosocial adjustment in children and adolescents with a craniofacial anomaly: diagnosis-specific patterns. Cleft Palate-Craniofacial Journal, 47(3), 264-272. doi:10.5555/08-227.1
Thamilselvan, P., Kumar, S. M., Murthy, J., Sharma, M. K., \& Kumar, R. N. (2015). Psychosocial issues of parents of children with cleft lip and palate in relation to their behavioral problems. Cleft Palate-Craniofacial Journal, 2(1), 53-57. doi:10.4103/0971-9261.55152

Wehby, G., Tyler, M. C., Lindgren, S., Romitti, P., Robbins, J., \& Damiano, P. (2012). Oral clefts and behavioral health of young children. Oral Diseases, 18(1), 74-84. doi:10.1111/j.16010825.2011.01847.x
Recebido: $29 / 07 / 2015$

$1^{a}$ revisão: 11/03/2016

$2^{a}$ revisão: 30/04/2016

Aceite final: $31 / 05 / 2016$ 